\title{
Do static sources respond to massive scalar particles from the Hawking radiation as uniformly accelerated ones do in the inertial vacuum?
}

\author{
J. Castiñeiras, ${ }^{*}$ I. P. Costa e Silva ${ }^{\dagger}$ and G. E. A. Matsas ${ }^{\ddagger}$ \\ Instituto de Física Teórica, Universidade Estadual Paulista, Rua Pamplona 145, 01405-900, São Paulo, SP, Brazil
}

(Received 18 November 2002; published 27 March 2003)

\begin{abstract}
We examine the recently found equivalence for the response of a static scalar source interacting with a massless Klein-Gordon field when the source is (i) static in Schwarzschild spacetime, in the Unruh vacuum associated with the Hawking radiation, and (ii) uniformly accelerated in Minkowski spacetime, in the inertial vacuum, provided that the source's proper acceleration is the same in both cases. It is shown that this equivalence is broken when the massless Klein-Gordon field is replaced by a massive one.
\end{abstract}

DOI: 10.1103/PhysRevD.67.067502

PACS number(s): 04.70.Dy, 04.62.+v

It was recently shown that the response $R^{S}\left(r_{0}, M\right)$ of a static scalar source with a radial coordinate $r_{0}$ outside a Schwarzschild black hole of mass $M$ interacting with massless scalar particles of Hawking radiation (associated with the Unruh vacuum) is exactly the same as the response $R^{M}\left(a_{0}\right) \equiv q^{2} a_{0} / 4 \pi^{2}$ of such a source when it is uniformly accelerated in the inertial vacuum of Minkowski spacetime, provided that the source's proper acceleration $a_{0}$ is the same in both cases [1]. (Here, $q$ is a coupling constant.) Now, according to the Fulling-Davies-Unruh (FDU) effect [2,3], the inertial vacuum in Minkowski spacetime corresponds to a thermal state as seen by uniformly accelerated observers confined to the Rindler wedge. Thus, the equivalence above can be rephrased by saying that the response of a static scalar source with some fixed proper acceleration coupled to a massless scalar field is the same when it interacts either (i) with the Hawking radiation associated with the Unruh vacuum in Schwarzschild spacetime or (ii) with the FDU thermal bath in Rindler spacetime. This came as a surprise because structureless static scalar sources can only interact with zero-frequency field modes. Such modes probe the global geometry of spacetime and are accordingly quite different in Schwarzschild and Rindler spacetimes. Moreover, since the response in Schwarzschild spacetime $R^{S}\left(r_{0}, M\right)$ was expected to depend on $r_{0}$ and $M$ separately, it is striking that these parameters should combine themselves precisely so that $R^{S}\left(r_{0}, M\right)=q^{2} a_{0}\left(r_{0}, M\right) / 4 \pi^{2}$, as found in Ref. [1]. The fact that such an equivalence is not trivial can be also seen by the fact that it is not verified when (i) the Unruh vacuum is replaced by the Hartle-Hawking vacuum [1], (ii) the black hole is endowed with some electric charge [4] or even (iii) when the massless Klein-Gordon field is replaced by the electromagnetic one [5]. A deeper understanding of why such an equivalence in the response is verified for massless Klein-Gordon fields is still lacking. While it may prove to be just a remarkable coincidence, we feel that the problem deserves further analysis.

In this paper we show that providing some mass to the Klein-Gordon field is enough to break the equivalence. The

\footnotetext{
*Electronic address: jcastin@ift.unesp.br

${ }^{\dagger}$ Electronic address: ivanpcs@ift.unesp.br

‡Electronic address: matsas@ift.unesp.br
}

main technical difficulty associated with the field quantization in Schwarzschild spacetime is related to the fact that the positive and negative frequency modes used to expand the quantum field cannot be expressed in terms of known special functions. Although for massless spin-0 and spin- 1 fields outside Reissner-Nördstrom black holes, the quantization of the low-energy sector admits an analytic treatment [1,4,5] (see also [6]), this is not the case for massive fields, for which a numerical analysis turns out to be required. Throughout this paper, we adopt natural units in which $c=G=\hbar=k_{B}=1$ and spacetime signature $(+---)$.

The Schwarzschild line element describing a black hole of mass $M$ can be written as [7]

$$
d s^{2}=f(r) d t^{2}-f(r)^{-1} d r^{2}-r^{2}\left(d \theta^{2}+\sin ^{2} \theta d \varphi^{2}\right),
$$

where $f(r) \equiv 1-2 M / r$.

Let us now consider a free Klein-Gordon field $\Phi\left(x^{\mu}\right)$ with mass $m$ in this background, described by the action

$$
S=(1 / 2) \int d^{4} x \sqrt{-g}\left[\nabla^{\mu} \Phi \nabla_{\mu} \Phi-m^{2} \Phi^{2}\right],
$$

where $g \equiv \operatorname{det}\left\{g_{\mu \nu}\right\}$. In order to quantize the field, we look for a complete set of positive-frequency solutions of the Klein-Gordon equation $\left(\square+m^{2}\right) u_{\omega l \mathrm{~m}}^{\alpha}=0$ in the form

$$
u_{\omega l \mathrm{~m}}^{\alpha}\left(x^{\mu}\right)=\sqrt{\frac{\omega}{\pi}} \frac{\psi_{\omega l}^{\alpha}(r)}{r} Y_{l \mathrm{~m}}(\theta, \varphi) e^{-i \omega t},
$$

where $l \geqslant 0, \mathrm{~m} \in[-l, l]$ and $\omega$ are the angular momentum and frequency quantum numbers, respectively. Because the Klein-Gordon equation is of second order, there will be in general two independent sets of normalizable solutions, here chosen to be incoming modes (i) from the horizon and (ii) from infinity labeled by $\alpha=\mathrm{I}$ and $\alpha=\mathrm{II}$, respectively. The factor $\sqrt{\omega / \pi}$ has been inserted for later convenience and $Y_{l \mathrm{~m}}(\theta, \varphi)$ are the spherical harmonics. As a consequence, $\psi_{\omega l}^{\alpha}(r)$ must satisfy

$$
\left[-f(r) \frac{d}{d r}\left(f(r) \frac{d}{d r}\right)+V_{\mathrm{eff}}(r)\right] \psi_{\omega l}^{\alpha}(r)=\omega^{2} \psi_{\omega l}^{\alpha}(r),
$$

where the effective scattering potential $V_{\text {eff }}(r)$ is given by 


$$
V_{\text {eff }}(r)=(1-2 M / r)\left[2 M / r^{3}+l(l+1) / r^{2}+m^{2}\right] .
$$

We note that close to and far away from the horizon we have $V_{\text {eff }}(r) \approx 0$ and $V_{\text {eff }}(r) \approx m^{2}$, respectively. Thus, the frequency of the modes $u_{\omega l \mathrm{~m}}^{\alpha}$ with $\alpha=\mathrm{I}$ and $\alpha=$ II will be constrained so that $\omega \geqslant 0$ and $\omega \geqslant m$, respectively. Now, it is convenient to recast Eq. (4) in a Schrödinger-like form. For this purpose, we define a new dimensionless coordinate $y$ $\equiv r / 2 M$ and perform the change of variable $y \rightarrow x \equiv y+\ln \mid y$ -1 , so that Eq. (4) becomes

$$
\left\{-d^{2} / d x^{2}+4 M^{2} V_{\text {efft }}[r(x)]\right\} \psi_{\omega l}^{\alpha}(x)=4 M^{2} \omega^{2} \psi_{\omega l}^{\alpha}(x) .
$$

We can expand the scalar field operator $\hat{\Phi}\left(x^{\mu}\right)$ in terms of annihilation $\hat{a}_{\omega l \mathrm{~m}}^{\alpha}$ and creation $\hat{a}_{\omega l \mathrm{~m}}^{\alpha \dagger}$ operators as usual:

$$
\hat{\Phi}\left(x^{\mu}\right)=\sum_{\alpha=\mathrm{I}, \mathrm{II}} \sum_{l=0}^{\infty} \sum_{\mathrm{m}=-l}^{l} \int_{0}^{\infty} d \omega\left[u_{\omega l \mathrm{~m}}^{\alpha}\left(x^{\mu}\right) \hat{a}_{\omega l \mathrm{~m}}^{\alpha}+\text { H.c. }\right],
$$

where $u_{\omega l \mathrm{~m}}^{\alpha}\left(x^{\mu}\right)$ are orthonormalized according to the KleinGordon inner product [8]. As a consequence, $\hat{a}_{\omega l \mathrm{~m}}^{\alpha}$ and $\hat{a}_{\omega l \mathrm{~m}}^{\alpha \dagger}$ satisfy $\left[\hat{a}_{\omega l \mathrm{~m}}^{\alpha}, \hat{a}_{\omega^{\prime} l^{\prime} \mathrm{m}^{\prime}}^{\alpha^{\prime} \dagger}\right]=\delta_{\alpha \alpha^{\prime}} \delta_{l l^{\prime}} \delta_{\mathrm{mm}^{\prime}} \delta\left(\omega-\omega^{\prime}\right)$ and the Boulware vacuum $|0\rangle$ is defined by $\hat{a}_{\omega l \mathrm{~m}}^{\alpha}|0\rangle=0$ for every $\alpha$, $\omega, l$ and $\mathrm{m}[9]$.

Now, let us consider a pointlike static scalar source lying at $\left(r_{0}, \theta_{0}, \varphi_{0}\right)$ and described by

$$
j\left(x^{\mu}\right)=(q / \sqrt{-h}) \delta\left(r-r_{0}\right) \delta\left(\theta-\theta_{0}\right) \delta\left(\varphi-\varphi_{0}\right),
$$

where $h=-f(r)^{-1} r^{4} \sin ^{2} \theta$ is the determinant of the spatial metric induced on the equal $t$ time hypersurface $\Sigma_{t}$ and $q$ is a small coupling constant. We will be interested in analyzing the behavior of this source, coupled to the Klein-Gordon field $\hat{\Phi}\left(x^{\mu}\right)$, via the interaction action

$$
\hat{S}_{I}=\int d^{4} x \sqrt{-g} j \hat{\Phi}
$$

when it is immersed in the Hawking radiation emitted from the black hole. All the calculations will be carried out at the tree level.

The total response, i.e., particle emission and absorption probabilities per unit proper time of the source, is given by

$$
R^{S} \equiv \sum_{\alpha=\mathrm{I}, \mathrm{II}} \sum_{l=0}^{\infty} \sum_{\mathrm{m}=-l}^{l} \int_{0}^{+\infty} d \omega R_{\omega l \mathrm{~m}}^{\alpha}
$$

where

$$
R_{\omega l \mathrm{~m}}^{\alpha} \equiv \tau^{-1}\left\{\left|\mathcal{A}_{\omega l \mathrm{~m}}^{\alpha} \mathrm{em}\right|^{2}\left[1+n^{\alpha}(\omega)\right]+\left|\mathcal{A}_{\omega l \mathrm{~m}}^{\alpha} \mathrm{abs}\right|^{2} n^{\alpha}(\omega)\right\}
$$

and $\tau$ is the total proper time of the source. Here $\mathcal{A}_{\omega l \mathrm{~m}}^{\alpha}$ em $\equiv\left\langle\alpha \omega l \mathrm{~m}\left|\hat{S}_{I}\right| 0\right\rangle$ and $\mathcal{A}_{\omega l \mathrm{~m}}^{\alpha}$ abs $\equiv\left\langle 0\left|\hat{S}_{I}\right| \alpha \omega l \mathrm{~m}\right\rangle$ are the emis- sion and absorption amplitudes, respectively, of Boulware states $|\alpha \omega l \mathrm{~m}\rangle$ and $n^{\alpha}(\omega)$ is defined below. It is not difficult to show that

$$
\begin{aligned}
\left|\mathcal{A}_{\omega l \mathrm{~m}}^{\alpha \quad \mathrm{em}}\right| & =\left|\mathcal{A}_{\omega l \mathrm{~m}}^{\alpha} \mathrm{abs}\right| \\
& =2 q \sqrt{\pi \omega f\left(r_{0}\right)} / r_{0}\left|\psi_{\omega l}^{\alpha}\left(r_{0}\right)\right|\left|Y_{l \mathrm{~m}}\left(\theta_{0}, \varphi_{0}\right)\right| \delta(\omega) .
\end{aligned}
$$

The $\delta(\omega)$ reflects the fact that our structureless static source (8) can only interact with zero-energy modes. By letting Eq. (12) in Eq. (11), we obtain, from Eq. (10),

$$
R^{S}=\left.\sum_{l=0}^{\infty} C_{l}\left(r_{0}\right) \omega\left|\psi_{\omega l}^{\mathrm{I}}\left(r_{0}\right)\right|^{2}\left[1+2 n^{\mathrm{I}}(\omega)\right]\right|_{\omega=0}
$$

with $C_{l}\left(r_{0}\right)=q^{2}(2 l+1) \sqrt{f\left(r_{0}\right)} /\left(2 \pi r_{0}^{2}\right)$. Here, we have used the summation formula for spherical harmonics [10], $\sum_{\mathrm{m}=-l}^{l}\left|Y_{l \mathrm{~m}}\left(\theta_{0}, \varphi_{0}\right)\right|^{2}=(2 l+1) / 4 \pi$, the fact that $\psi_{\omega l}^{\mathrm{II}}$ vanishes for $\omega<m$ and $\tau=2 \pi f^{1 / 2}\left(r_{0}\right) \lim _{\omega \rightarrow 0} \delta(\omega)$ (see Refs. [1] and [4]).

In the Boulware vacuum, static observers see no particles at all and so $n^{\alpha}(\omega)=0$. Thus, since $\psi_{0 l}^{\mathrm{I}}\left(r_{0}\right)$ is finite for $r_{0}$ $>2 M$ (see below), it is straightforward from Eq. (13) that the response of our source vanishes in this case. [The precise form of $\psi_{0 l}^{\mathrm{I}}\left(r_{0}\right)$ is only numerically available but close and far away from the horizon it can be inferred analytically from Eq. (15).] In the Unruh vacuum, however, this is not so. The Unruh vacuum corresponds [8] to a thermal flux radiated away from the horizon at temperature $\beta^{-1}=1 / 8 \pi M[11]$ as measured by asymptotic static observers and hence $n^{\mathrm{I}}(\omega)$ $\equiv\left(e^{\omega \beta}-1\right)^{-1}$ and $n^{\mathrm{II}}=0$. In the presence of a background thermal bath, the absorption and stimulated emission rates will lead in general to a non-zero response. This is only possible because the thermal factor $\left(e^{\omega \beta}-1\right)^{-1}$ diverges as $\omega^{-1} / \beta$ when $\omega \rightarrow 0$. Thus, in the Unruh vacuum case, on which we focus, we need a "regulator" to avoid the appearance of intermediate indefinite results " $0 \times \infty$ " (for a more comprehensive discussion on the interaction of static sources with zero-energy modes, see Ref. [12]). For this purpose, we let the coupling constant $q$ oscillate with frequency $\omega_{0}$ by replacing $q$ by $q_{\omega_{0}} \equiv \sqrt{2} q \cos \left(\omega_{0} t\right)$ in Eq. (8) and taking the limit $\omega_{0} \rightarrow 0$ at the end of our calculations. The factor $\sqrt{2}$ has been introduced to ensure that the time average $\left\langle\left|q_{\omega_{0}}(t)\right|^{2}\right\rangle_{t}$ $=q^{2}$ since the absorption and emission rates are functions of $q^{2}$. Other equivalent regularization procedures can be devised [13]. A straightforward calculation with the oscillating source [4] gives

$$
R^{S}\left(r_{0}, M\right)=\frac{q^{2} f\left(r_{0}\right)^{1 / 2}}{16 \pi^{2} M r_{0}^{2}} \lim _{\omega_{0} \rightarrow 0} \sum_{l=0}^{\infty}(2 l+1)\left|\psi_{\omega_{0} l}^{\mathrm{I}}\left(r_{0}\right)\right|^{2} .
$$

Note that only $\alpha=\mathrm{I}$ appears in Eq. (14). This can be seen as reflecting the fact that the Unruh vacuum corresponds to a thermal flux of particles being radiated only from the horizon. It should be noticed, however, that the same response (14) holds when we replace the Unruh with the HartleHawking vacuum. This is so because the extra thermal flux 


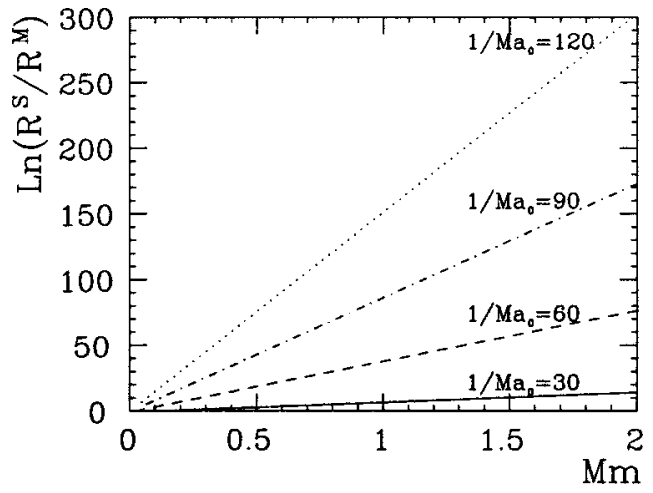

FIG. 1. $\operatorname{Ln}\left(R^{S} / R^{M}\right)$ is plotted as a function of $m$ (where, for the sake of convenience, we have used the black hole mass $M$ as a standard scale). The equality between $R^{S}$ and $R^{M}$ is recovered when $m \rightarrow 0$, as expected, but not for general values of $m$. [In plotting this graph, we have summed up to $l=18$ in Eq. (14).]

coming from infinity in the Hartle-Hawking vacuum (which should be considered in addition to the one coming from the horizon) is composed of particles with frequency $\omega \geqslant m$ [see discussion below Eq. (5)]; i.e., this extra thermal flux is not populated with zero-energy particles which are the only ones which can interact with our source.

In order to compute $R^{S}$ in Eq. (14), we shall evaluate $\psi_{\omega_{0} l}^{\mathrm{I}}$ (with $\omega_{0} \rightarrow 0$ ) numerically. By using Eq. (6), it is easy to see that $\psi_{\omega l}^{\mathrm{I}}(x)$ has the following asymptotic forms when $\omega$ $<m$ :

$$
\psi_{\omega l}^{\mathrm{I}}(x) \approx \begin{cases}A_{\omega l}\left(e^{2 i M \omega x}+\mathcal{R}_{\omega l} e^{-2 i M \omega x}\right) & (x \ll-1), \\ B_{\omega l} e^{-2 M \sqrt{m^{2}-\omega^{2} x}} & (x \gg 1),\end{cases}
$$

where $A_{\omega l}$ and $B_{\omega l}$ are constants and $\left|\mathcal{R}_{\omega l}\right|^{2}=1$, which is calculated by using Eq. (15) in Eq. (6). For $\omega \approx 0$ it can be shown that $\mathcal{R}_{\omega l} \approx-1+\mathcal{O}(\omega)$ (see [1] and [4] for a derivation in the massless case). Indeed, this solution describes modes that leave the horizon, "scatter off the geometry" and fall back to the horizon. The normalization constant $A_{\omega l}$ $=(2 \omega)^{-1}$ is analytically obtained (up to an arbitrary phase) by demanding that the normal modes (3) be orthonormalized with respect to the Klein-Gordon inner product (see, e.g., Ref. [1] for details). $B_{\omega l}$ is obtained numerically and is finite for $\omega \geqslant 0$. The modes $\psi_{\omega l}^{\mathrm{I}}$ can be obtained numerically for small $\omega / m$ and different $l$ values by evolving Eq. (6) with the effective potential (5) and "boundary conditions" (15). The corresponding total response $R^{S}$ can be obtained, then, from Eq. (14). We note that the larger the value of $l$, the higher the barrier of the scattering potential $V_{\text {eff }}(r)$ [14] and therefore the main contributions come from modes with small $l$. How far we must sum over $l$ in Eq. (14) to obtain a satisfactory numerical result will depend on how close to the black hole horizon the source lies. The closer to the horizon, the further over $l$ we must sum. We have checked our numerical code for $m=0$ where the response is known analytically [1].

Our results for $R^{S}$ will be exhibited in comparison with the response $R^{M}$ obtained when our scalar source is uni-

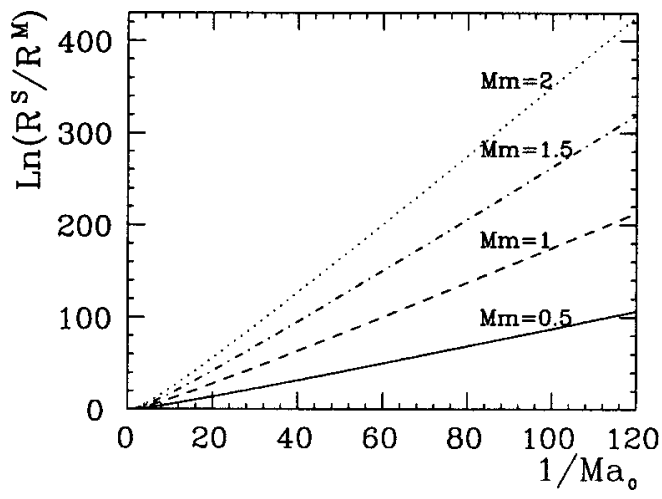

FIG. 2. In this figure, it becomes clear how the equality between $R^{S}$ and $R^{M}$ is broken for various masses of the Klein-Gordon field. Note that the more we move the source away from the horizon (which corresponds to decreasing $a_{0}$ ), the more $R^{S}$ deviates from $R^{M}$. On the other hand, $R^{S} \rightarrow R^{M}$ as we approach the horizon $\left(a_{0}\right.$ $\rightarrow \infty$ ) (see discussion in the text). [In plotting this graph, we have summed up to $l=18$ in Eq. (14).]

formly accelerated (with proper acceleration $a_{0}$ ) in the usual inertial vacuum in Minkowski spacetime. $R^{M}$ can be equivalently computed either with respect to inertial or uniformly accelerated observers. We favor the latter here. Accordingly, we shall perform the quantization of the massive KleinGordon field in the Rindler wedge, which can be described by the line element [8]

$$
d s^{2}=e^{2 a_{0} \xi}\left(d \tau^{2}-d \xi^{2}\right)-d x^{2}-d y^{2}
$$

with $-\infty<\tau, \xi, x, y<+\infty$. The corresponding Klein-Gordon orthonormalized positive-frequency modes are

$$
\begin{aligned}
u_{\omega \mathbf{k}_{\perp}}\left(x^{\mu}\right)= & \frac{\sqrt{\sinh \left(\pi \omega / a_{0}\right)}}{2 \pi^{2} a_{0}^{1 / 2}} K_{i \omega / a_{0}}\left[\frac{\sqrt{\mathbf{k}_{\perp}^{2}+m^{2}} e^{a_{0} \xi}}{a_{0}}\right] \\
& \times e^{i \mathbf{k}_{\perp} \cdot \mathbf{x}_{\perp}-i \omega \tau},
\end{aligned}
$$

where $K_{\nu}(x)$ is the modified Bessel function [10], $\mathbf{x}_{\perp}$ $=(x, y)$ and $\mathbf{k}_{\perp} \equiv\left(k_{x}, k_{y}\right)$ denotes the momentum transverse to the direction of acceleration. In these coordinates, our source with constant proper acceleration $a_{0}$ will be described by $j\left(x^{\mu}\right)=q \delta(\xi) \delta(x) \delta(y)$. The total response is given, in this case, by

$$
R^{M} \equiv \int d \mathbf{k}_{\perp}^{2} \int_{0}^{+\infty} d \omega R_{\omega \mathbf{k}_{\perp}}
$$

where $R_{\omega \mathbf{k}_{\perp}} \equiv \tau^{-1}\left\{\left|\mathcal{A}_{\omega \mathbf{k}_{\perp}}^{\mathrm{em}}\right|^{2}[1+n(\omega)]+\left|\mathcal{A}_{\omega \mathbf{k}_{\perp}}^{\text {abs }}\right|^{2} n(\omega)\right\}$. Here $\mathcal{A}_{\omega \mathbf{k}_{\perp}}^{\mathrm{em}} \equiv\left\langle\omega \mathbf{k}_{\perp}\left|\hat{S}_{I}\right| 0\right\rangle$ and $\mathcal{A}_{\omega \mathbf{k}_{\perp}}^{\mathrm{abs}} \equiv\left\langle 0\left|\hat{S}_{I}\right| \omega \mathbf{k}_{\perp}\right\rangle$ are the emission and absorption amplitudes, respectively, of Rindler states $\left|\omega \mathbf{k}_{\perp}\right\rangle$ and $n(\omega)=1 /[\exp (\beta \omega)-1]$, where $\beta^{-1}=a_{0} / 2 \pi$ is the temperature of the FDU thermal bath associated with the inertial vacuum as measured by the Rindler observer lying at $\xi=0$. The response can be shown to be

$$
R^{M}\left(a_{0}\right)=\frac{q^{2} a_{0}}{2 \pi^{2}} \int_{0}^{\infty} d x x K_{0}^{2}\left[\sqrt{x^{2}+\left(m / a_{0}\right)^{2}}\right] .
$$


In Fig. 1 we plot $R^{S}\left(a_{0}, M\right) / R^{M}\left(a_{0}\right)$ as a function of the scalar field mass $m$ for different $a_{0}$ 's. We recall that the source's proper acceleration $a_{0}$ is a one-to-one function of its radial position $r_{0}: a_{0}=M /\left(r_{0}^{2} \sqrt{1-2 M / r_{0}}\right)$. We note that in general $R^{S}\left(a_{0}, M\right) \neq R^{M}\left(a_{0}\right)$. The equivalence is only recovered when $m \rightarrow 0$.

In Fig. 2 we display how the equivalence is broken for various values of $m$. Here $R^{S}$ deviates from $R^{M}$ as one goes away from the horizon at a rate depending on $m$. Note that indeed $R^{S}$ approaches $R^{M}$ as $m$ goes to zero. The fact that $R^{S} / R^{M} \rightarrow 1$ in the massive case for $a_{0} \rightarrow \infty$ could be analytically predicted from the equivalence found in the massless case as follows. On the one hand, close to the black hole horizon, the potential (5) is not significantly influenced by the mass of the field. As a consequence, in this region, massive and non-massive outgoing modes $\psi_{\omega l}^{\mathrm{I}}$, which are the relevant ones in Eq. (14), behave similarly. Thus, it is expected that for sources close enough to the horizon, i.e. $a_{0} / m \gg 1, R^{S}$ should be approximately the same for massive and non-massive fields. On the other hand, it is easy to see from Eq. (19) that the same statement is true for $R^{M}$. Hence the equivalence found for the massless case also implies that $R^{S} / R^{M} \rightarrow 1$ for massive fields as long as $a_{0} / m \rightarrow \infty$.
We have shown in this paper that the response of a static scalar source with some fixed proper acceleration coupled to a massive scalar field is not the same when it interacts (i) with the Hawking radiation in Schwarzschild spacetime and (ii) with the FDU thermal bath in Rindler spacetime. This reinforces how unexpected was the equivalence found for the responses in the massless case, since the normal modes depend in general on the global structure of the underlying spacetime. Despite our best efforts we have found no special features in the massless Klein-Gordon field suggesting that the equivalence found in this case was to be expected. In particular, we emphasize that there is no equivalence [5] when the massless scalar particles are replaced by photons (which are also massless). Thus, any eventual explanation would have to deal not only with the mass but also with the spin of the field. A deeper understanding of this issue would be welcome.

J.C. and I.C. would like to acknowledge full support from Fundação de Amparo à Pesquisa do Estado de São Paulo. G.M. is thankful to Conselho Nacional de Desenvolvimento Científico e Tecnológico and Fundação de Amparo à Pesquisa do Estado de São Paulo for partial support.
[1] A. Higuchi, G.E.A. Matsas, and D. Sudarsky, Phys. Rev. D 56, R6071 (1997); 58, 104021 (1998).

[2] W.G. Unruh, Phys. Rev. D 14, 870 (1976).

[3] S.A. Fulling, Phys. Rev. D 7, 2850 (1973); P.C.W. Davies, J. Phys. A 8, 609 (1975).

[4] J. Castiñeiras and G.E.A. Matsas, Phys. Rev. D 62, 064001 (2000).

[5] L.C.B. Crispino, A. Higuchi, and G.E.A. Matsas, Phys. Rev. D 58, 084027 (1998); 63, 124008 (2001).

[6] G. Cognola and P. Lecca, Phys. Rev. D 57, 1108 (1998).

[7] R.W. Wald, General Relativity (University of Chicago Press, Chicago, 1984).

[8] N.D. Birrell and P.C.W. Davies, Quantum Field Theory in Curved Space (Cambridge University Press, Cambridge, England, 1982); S.A. Fulling, Aspects of Quantum Field Theory in Curved Space-Time (Cambridge University Press, Cam- bridge, England, 1989)

[9] D.G. Boulware, Phys. Rev. D 11, 1404 (1975); 12, 350 (1975).

[10] I.S. Gradshteyn and I.M. Ryzhik, Table of Integrals, Series and Products (Academic Press, New York, 1980); Handbook of Mathematical Functions, edited by M. Abramowitz and I.A. Stegun (Dover, New York, 1965).

[11] S.W. Hawking, Nature (London) 248, 30 (1974); Commun. Math. Phys. 43, 199 (1975); J.B. Hartle and S.W. Hawking, Phys. Rev. D 13, 2188 (1976).

[12] A. Higuchi, G.E.A. Matsas, and D. Sudarsky, Phys. Rev. D 45, R3308 (1992); 46, 3450 (1992); H. Ren and E.J. Weinberg, ibid. 49, 6526 (1994).

[13] D.E. Díaz and J. Stephany, Class. Quantum Grav. 19, 3753 (2002).

[14] J. Castineiras, L.C.B. Crispino, G.E.A. Matsas, and D.A.T. Vanzella, Phys. Rev. D 65, 104019 (2002). 